\title{
Berberine-loaded Janus gold mesoporous silica nanocarriers for chemo/radio/photothermal therapy of liver cancer and radiation-induced injury inhibition
}

This article was published in the following Dove Press journal:

International Journal of Nanomedicine

\author{
Xiao-Dong $\mathrm{Li}^{1-3}$ \\ Zheng Wang ${ }^{1,2}$ \\ Xin-Rui Wang ${ }^{4}$ \\ Dan Shao' \\ Xi Zhang ${ }^{5}$ \\ $\mathrm{Li} \mathrm{Li}$ \\ Ming-Feng $\mathrm{Ge}^{\prime}$ \\ Zhi-Min Chang' \\ Wen-Fei Dong'
}

'CAS Key Laboratory of Bio-Medical Diagnostics, Suzhou Institute of

Biomedical Engineering and Technology,

Chinese Academy of Sciences, Suzhou

215163, People's Republic of China;

${ }^{2}$ University of Chinese Academy of

Sciences, Beijing 100049, People's

Republic of China; ${ }^{3}$ Department of

Echocardiography, The First Hospital of Jilin University, Changchun I3002I,

People's Republic of China; ${ }^{4}$ Department of Hepatology, The First Hospital of Jilin University, Changchun I3002I, People's Republic of China; ${ }^{5}$ Department of Rheumatology, Third Affiliated Hospital of Sun Yat-sen University, Guangzhou 510630, People's Republic of China

Correspondence: Zhi-Min Chang; Wen-Fei Dong

CAS Key Laboratory of Bio-Medical Diagnostics, Suzhou Institute of Biomedical Engineering and Technology, Chinese Academy of Sciences, 88 Keling Road, Suzhou 215163, People's Republic of China

Tel +86 5I2 5I85593I;

+8651269588307

Email changzm@sibet.ac.cn;

wenfeidong@sibet.ac.cn
Background: The combination of chemotherapy with radiotherapy serves as a common therapeutic strategy in clinics. However, it is unsatisfactory due to its poor therapeutic efficiency and severe side-effects originating from chemotherapy-exerted systemic toxicity as well as radiation-induced injury.

Purpose: Hence, Berberine (Ber), an isoquinolin alkaloid with low toxicity and protective effects against radiotherapy, was used as a novel chemotherapeutic agent for chemo-radiotherapy of liver cancer.

Patients and methods: We preloaded Ber into folic acid targeting Janus gold mesoporous silica nanocarriers (FA-JGMSNs) for overcoming the poor bioavailability of Ber. Furthermore, FA-JGMSNs were not only employed as radiosensitizers for expanding radiotherapeutic effect, but also used as photothermal agents for supplementing chemo-radiotherapeutic effect by local photothermal therapy.

Results: In vitro and in vivo experiemtal results demonstrated the highly efficient antitumor effect, good biosafety as well as the effective protection of normal tissue of this nanoplatform.

Conclusion: Based on its superb performance, we believe our work provided a feasible strategy for triple-therapies of liver cancer.

Keywords: Berberine, chemotherapy, radio-sensitization, Au MSNs, Janus, photothermal therapy

\section{Introduction}

Liver cancer is considered to be one of the most common and formidable diseases. ${ }^{1-3}$ Chemotherapy and radiotherapy (RT) are standard therapeutic methods in clinic and the combination of chemotherapy with radiotherapy has been demonstrated to exert a synergistic anti-tumor effect. ${ }^{4,5}$ However, this combined chemo-radiotherapy in clinic is unsatisfactory due to its severe side-effects and limited therapeutic outcomes. ${ }^{6-8}$ During RT, ionization radiation inevitably passes through peritumoral normal tissues, generating a series of side-effects. ${ }^{9}$ Chemotherapeutic drugs further aggravate the adverse effects due to their highly systemic toxicities and the lack of specificity. ${ }^{10,11}$ In addition, multi-drug resistance and radio-resistance also limit the outcomes of chemo-radiotherapy. ${ }^{12}$ Hence, it is urgent to develop new chemo-radiotherapeutic strategies for highly efficient and safe treatment of liver cancer. 
Berberine (Ber), an isoquinolin alkaloid isolated from natural herbs, has exhibited a remarkable anticancer activity in recent researches. ${ }^{13,14}$ More importantly, Ber possesses fewer side-effects and resistance compared with traditional chemotherapeutic drugs and can significantly reduce radiationinduced organic injury. ${ }^{15-17}$ Hence, Ber combined with RT would surmount the severe side-effects of chemoradiotherapy. However, the application of Ber is limited because of its poor aqueous solubility, leading to reduced bioavailability. ${ }^{18-20}$ Moreover, this chemo-radiotherapy is insufficient to eradicate tumor tissues. Utilizing high- $Z$ nanomaterials as radiosensitizers can effectively impove ionizing radiation delivery in the tumor site and achieve a higher radiotherapeutic outcome. ${ }^{21-23}$ Additionally, employing photothermal therapy (PTT) as an adjuvant treatment of chemoradiotherapy has also attracted great attention owing to the safe therapeutic modality of PTT and its excellent capacity to potentiate chemotherapeutic and radiotherapeutic effects by influencing cancer cell metabolism and changing the microenvironment of tumor. ${ }^{24-27}$ Thus, the integration of Ber therapy, radiotherapy sensitization and PTT would be a feasible method for simultaneously achieving a powerful therapeutic effect and low side-effects as well as a protective effect to normal tissues. However, it still remains challenging to seek a proper agent for synchronously driving the triple-modal therapies.

The thriving nanotechnology field has brought out new opportunities for highly efficient, specific and personalized cancer theranostics. ${ }^{28-30}$ Among them, gold-based nanoplatforms have drawn great attentions because of their outstanding properties and applications such as tunable surface plasmon resonance properties for PTT, strong X-ray attenuation for computed tomography(CT) imaging contrasts as well as high $\mathrm{Z}$ number for radiotherapy sensitization. ${ }^{31-33}$ Recently, gold mesoporous silica nanomaterials exhibited superior advantages on cancer multimodel therapies owing to their good biocompatibilities, high surface area, easily modified surface as well as multiple physical and chemical properties. ${ }^{34-36}$ Our group previously designed uniform Janus gold mesoporous silica nanocarriers (JGMSNs). These JGMSNs have been demonstrated to be not only superior radiosensitizers and CT imaging contrasts for CT imaging-mediated radiotherapy, but also intriguing drug vehicles because of their strong drug loading capacity and responsive drug release properties. ${ }^{37}$ Additionally, JGMSNs also show high photothermal conversion efficiency as photothermal agents for local PTT. More importantly, the asymmetric structure of
JGMSNs facilitates them to harness multiple functionalities without interference, thus exerting a maximal synergistic effect. ${ }^{38}$ Hence, JGMSNs would be proper candidates for loading Ber and realizing the triple-modal therapy of liver cancer. Folic acid (FA) is one of the most effective targeting ligands because excessive folate receptors exist on most of the liver cancer membranes. ${ }^{39-43}$ Hence, in this present study, FA was conjugated on the surface of JGMSNs (FAJGMSNs) for improving the target to liver cancer cells and Ber was preloaded into FA-JGMSNs (FA-JGMSNs-Ber) via surface-decorated carboxyl groups for tumor microenvironment-responsive release (Scheme 1). Then, we investigated the enhanced Ber therapy, radiation sensitization as well as PTT of FA-JGMSNs-Ber and evaluated the combined therapeutic effects and biosafety of this triple-model therapy in vitro and in vivo experiments. More importantly, the excellent protective effect of FA-JGMSNs-Ber against radiation-induced injury was demonstrated. Our work revealed the feasibility of these nanocarriers on the delivery of Ber for achieving high-effcient and safe triple-modal therapies of liver cancer.

\section{Methods}

\section{Preparation of FA-JGMSNs-Ber and characterization}

JGMSNs were synthesized according to our previous reports. The morphology of JGMSNs was characterized by transmission electron microscopy (TEM, JEM-2100; JEOL). To prepare FA-targeting JGMSNs, $2 \mathrm{mg}$ of FApoly (ethylene glycol) (PEG)-N-hydroxy succinimide (NHS) was added into $10 \mathrm{~mL}$ of the amino-modification JGMSN solution $(1 \mathrm{mg} / \mathrm{mL})$. After ultrasonic treatment and stirred overnight at room temperature, FA-JGMSNs were obtained by centrifugation. The UV-visible (vis)-NIR absorption spectra of free FA, gold nanorod, JGMSNs and FA-JGMSNs were measured by a U-3310 spectrophotometer (Hitachi, Tokyo, Japan). The size distribution of JGMSNs and FA-JGMSNs were measured by a Nano ZS 90 Nano-sizer (Malvern Instruments Ltd., Malvern, Worcestershire, UK). The mesoporous structure of FAJGMSNs were detected by the Brunauer-Emmett-Teller (BET) method and the Barrett-Joyner-Halenda method. To Preload Ber, we injected $10 \mathrm{~mL}$ alcohol solution of Ber $(0.5 \mathrm{mg} / \mathrm{mL})$ into $10 \mathrm{~mL}$ of FA-JGMSNs solution $(1 \mathrm{mg} / \mathrm{mL})$. Then, the mixture was stirred for $12 \mathrm{~h}$ and FAJGMSNs-Ber were collected and washed three times with PBS. Next, we collected the supernatant and detected the 


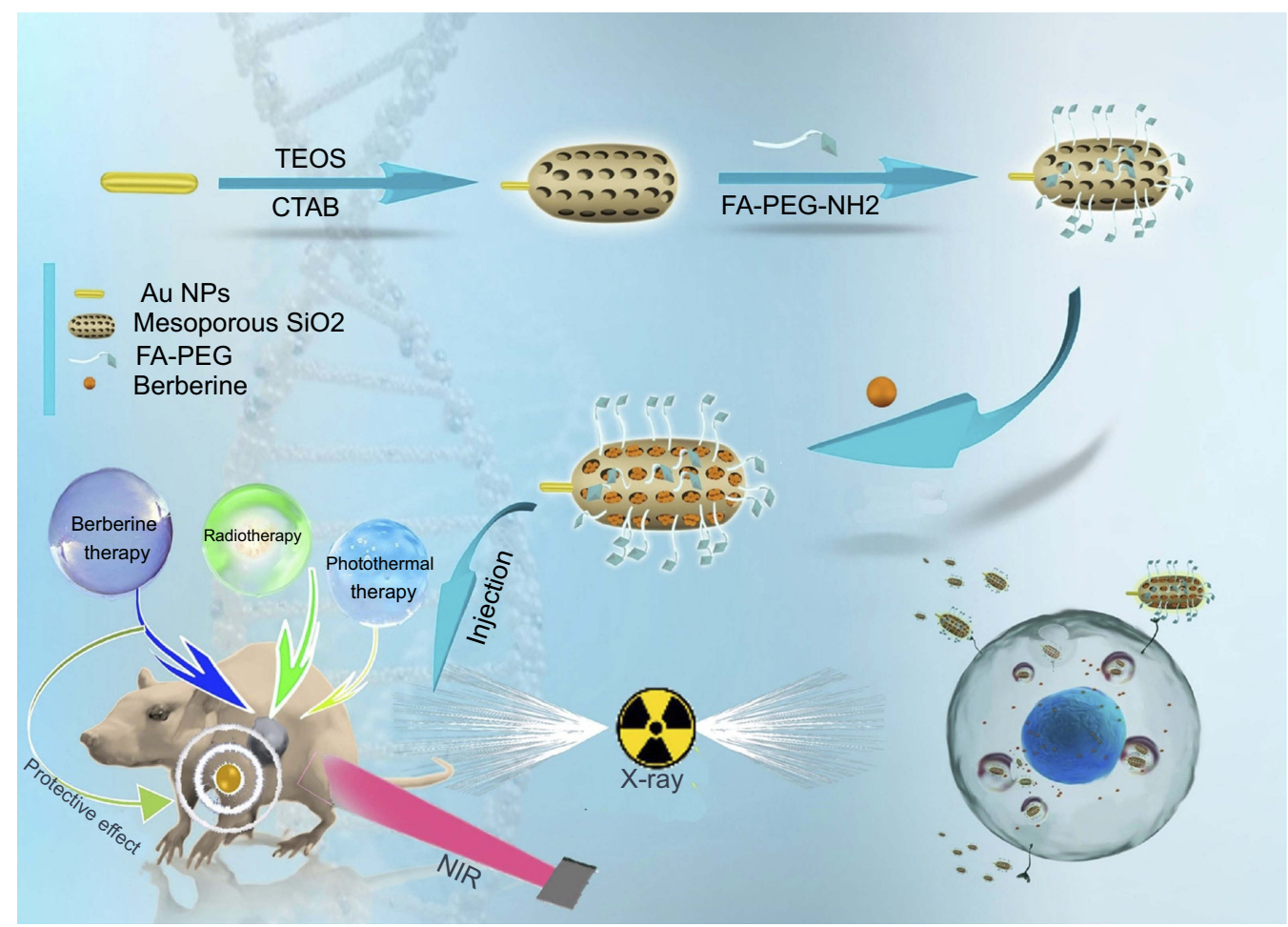

Scheme I Janus gold mesoporous silica nanocarriers loading Berberine for chemo/radio/photothermal therapy of liver cancer and radiation-induced injury inhibition.

quantity of Ber by UV-visible spectrophotometry of 480 $\mathrm{nm}$. The Ber loading efficiency and Ber loading content were calculated by the equations 1) Loading efficiency (\%) $=$ mass of Ber in JGMSNs/initial mass of drug; and 2) loading content $(\%)=$ mass of mass of Ber in JGMSNs/ mass of JGMSNs-Ber. To measure the release of Ber, $5 \mathrm{mg}$ of FA-JGMSNs-Ber were encapsulated into a dialysis bag in the $10 \mathrm{~mL}$ of PBS solutions with different $\mathrm{pH}$ values (pH 7.4 or 5.5). The release of Ber was measured by UVvisible spectrophotometry.

\section{Cell uptake of nanocarriers and intracellular Ber release}

SMMC-7721 cells and HL-7702 cells were cultured in a 24-well plate $\left(2 \times 10^{4}\right.$ cells per well). Then, FITClabeled FA-JGMSNs or JGMSNs $(10 \mu \mathrm{g} / \mathrm{mL})$ were added into these cells and co-incubated for 3 hours. In the free FA block group, $1 \mathrm{Mm}$ FA was co-incubated with FAJGMSNs. Next, these cells were washed, trypsinized and resuspended for flow cytometry measurements (FACS; BD Biosciences, Franklin Lakes, NJ, USA). To measure the intracellular Ber release of DOX, $12.5 \mu \mathrm{g} / \mathrm{mL}$ of FAJGMSNs-Ber or JGMSNs-Ber or equal amount of Ber $(2.5 \mu \mathrm{g} / \mathrm{mL})$ were co-incubated with these cells. After 3 hours, we stained the nuclei with Hoechst 33,258 for 5 minutes and detected the Ber using the CLSM (Olympus FV1000, Tokyo, Japan). We also incubated FA-JGMSNsBer or JGMSNs-Ber with SMMC-7721 cells for 3, 6 or 12 hours. Then, these cells were washed with PBS twice and allowed to excrete Ber for another 6 or 12 hours. Next, these cells were washed again and the intracellular Ber were measured by FACS.

\section{Cytotoxicity assessment}

Human liver cancer cell line SMMC-7721 cells and human normal liver cell line HL-7702 cells were cultured into a 96well plates $\left(5 \times 10^{3}\right.$ cells per well) overnight. No ethics committee permission was required for the use of SMMC-7721 cells and HL-7702 cells because only certificated cell lines provided by ATCC were used. Then, FA-JGMSNs-Ber, JGMSNs-Ber, FA-JGMSNs, JGMSNs or Ber at various concentration were respectively co-incubated with these cells for 24 hours. In the same parallel groups, the dose of free Ber was equal to the weight of Ber in FA-JGMSNs-Ber or JGMSNs-Ber. Moreover, the dose of FA-JGMSNs or JGMSNs was equal to the weight of FA-JGMSNs-Ber or JGMSNs-Ber removing the weight of Ber in nanocarriers. The weight of Ber in nanocarriers were calculated by the Ber loading content of these nanocarriers. Then, the cell 
viabilities were measured by traditional SRB assays. To detect the radiation sensitization and chem-radiotherapies, 5 Gy of X-ray irradiation ( $1 \mathrm{~Gy} / \mathrm{min})$ was used to irradiate cells after various treatments. The radiation was carried out by $6 \mathrm{MV}$ irradiation beams (TrueBeam; Varian Medical System, Palo Alto, CA, USA). To measure the photothermal effect, the NIR-treated groups were exposed under an 808 $\mathrm{nm}$ laser for 5 minutes $\left(1 \mathrm{~W} \mathrm{~cm}^{-2}\right)$ after various treatments.

\section{In vivo antitumor effect and safety} assessments

Approximately $20 \mathrm{~g}$ of male nude mice were purchased from the Experimental Animal Center of Jilin University and were maintained in a conventional animal housing facility. All animal experimental protocols were approved by the Ethics Committee for the Use of Experimental Animals of Jilin University. The experimental manipulation of mice was conducted in accordance with the National Institute of Health Guide for the Care and Use of Laboratory Animals and the approval of the Scientific Investigation Board of Science and Technology of Jilin Province (Changchun, People's Republic of China). These mice were injected with $5 \times 10^{6}$ SMMC-7721 cells in the right shoulder to establish xenografts models. When the tumor volumes reached to approximately $80-100 \mathrm{~mm}^{3}$, these mice were randomly divided into eights groups ( $\mathrm{n}=3$ /group). PBS, FA-JGMSNs (20 mg/kg), JGMSNs-Ber $(25 \mathrm{mg} / \mathrm{kg})$ and FA-JGMSNs-Ber $(25 \mathrm{mg} / \mathrm{kg})$ were intravenously injected and free Ber $(5 \mathrm{mg} / \mathrm{kg})$ was intraperitoneally injected into the mice every 3 days. In X-ray irradiation groups, $1 \mathrm{~Gy} / \mathrm{min}$ of X-ray radiation with a $1.5 \mathrm{~cm} \times 1.5 \mathrm{~cm}$ radiation field was used to irradiate the entire tumor for 5 minutes after 1 day of post-injections. In NIR irradiation groups, these tumor sites of the mice were exposed under an $808 \mathrm{~nm}$ laser $\left(1 \mathrm{Wcm}^{-2}\right)$ for 5 minutes at 24-hour and 48 hour administration. The tumor volume was measured and calculated by an equation: volume $=$ length $\times$ widt $^{2} \times 0.52$. At 23 days, all the mice were sacrificed. The tumor was harvested to weigh and the serum was collected for detecting the levels of alanine aminotransferase (ALT), aspartate aminotransferase (AST), blood urea nitrogen (BUN), creatinine (CRE).

\section{The protective effect against}

\section{radiation-induced intestinal injury}

Approximately $20 \mathrm{~g}$ of nude mice bearing SMMC-7721 xenograft were randomized into five groups and treated with PBS, free Ber (5 mg/kg), FA-JGMSN (20 mg/kg) and FA-JGMSNs-Ber $(25 \mathrm{mg} / \mathrm{kg})$ every 3 days. Then, $1 \mathrm{~Gy} /$ min of X-ray radiation was used to irradiate the entire tumor for $0,5,10$ minutes after 1 day of post-injections. After 3 times RT, the body weight and the average intake of food were measured. Then, the serum was collected and intestinal fatty acid-binding protein (iFABP), and diamine oxidase (DAO) were measured by an assay kit.

\section{Statistical analyses}

All the data represented three separate experiments and presented as the mean $\pm \mathrm{SD}$. The different groups were analyzed by a one-way analysis of variance. Bonferroni post hoc test was implemented for the analyses of different groups. $P<0.05$ represented a statistically significant difference.

\section{Results and discussions}

JGMSNs were synthesized by a modified sol-gel method as previous reports. ${ }^{44}$ As shown in Figure 1A, JGMSNs exhibited an asymmetric structure, which consist of a gold nanorod with dimensions of 50-60/10-15 nm and a silica stick with dimensions of 200-250/100-120 nm. To improve the stability of these JGMSNs and endow them with liver cancer-targeting capability, JGMSNs was functionalized by carboxyl groups and then FA-PEG was conjugated on the surface of JGMSNs to form FA-JGMSNs. The FT-IR spectra in Figure S1 exhibited that the characteristic adsorption peaks of FA-PEG-NH $\mathrm{NH}_{2}$ at $1335^{-1}$ and $1100 \mathrm{~cm}^{-1}$ appeared in the FA-JGMSNs group, while a new adsorption peaks of the amide carbonyl groups at $1640^{-1}$ and $1540 \mathrm{~cm}^{-1}$ were also observed in the FAJGMSNs group, demonstrating that FA was conjugated on the surface of these nanoparticles. Furthermore, The UV-visible spectra in Figure 1B showed an absorption peak of approximately $280 \mathrm{~nm}$ which was consistent with the characteristic absorption peak of FA was located in the FA- JGMSNs, also confirming the successful conjugation of the FA. Additionally, FA-JGMSNs possessed an absorption peak of $740 \mathrm{~nm}$, which was a slight red shift in comparison to that of Au rods, suggesting that the FAJGMSNs preserved the optical properties of $\mathrm{Au}$ rods. Furthermore, the particle sizes of FA-JGMSNs remained constant for 5 days, whereas JGMSNs aggregated, suggesting that PEGylation process improved the long-term stability of JGMSNs (Figure S2). Then, we investigated the mesoporous structure of FA-JGMSNs by BET method and BJH method. The N2 adsorption-desorption isotherms in Figure 1C showed type IV curves, confirming the 

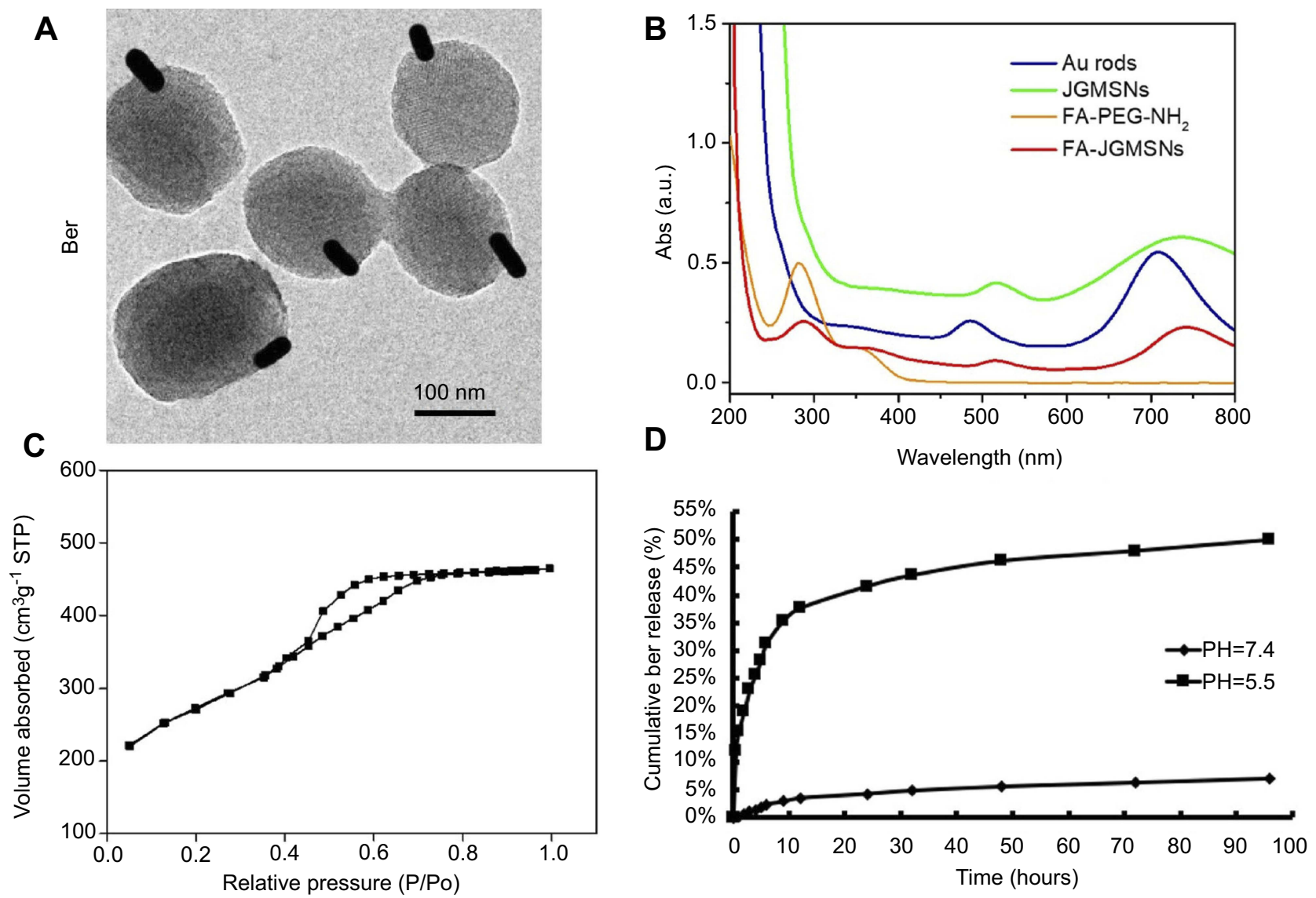

Figure I Characterization of FA-JGMSNs: (A) TEM images, (B) UV-vis absorption spectra, (C) $\mathrm{N}_{2}$ adsorption/desorption isotherms of FA-JGMSNs, and (D) Drug release behavior of FA-JGMSNs-Ber at different $\mathrm{pH}$ values as a function of cumulative time.

existence of mesopores. The average mesopore size of FAJGMSNs was $2.2 \mathrm{~nm}$ (Figure S3), and its BET surface area and total pore volume were $723.6 \mathrm{~m}^{2} / \mathrm{g}$ and $0.46 \mathrm{~cm}^{3} / \mathrm{g}$, respectively. The excellent mesoporous properties of FAJGMSNs indicated their high Ber loading capacity. For Ber loading, we dissolved Ber into dimethyl sulphoxide (DMSO) for increasing its solubility. Then, Ber was preloaded into the silica body of FA-JGMSNs via the charge interaction. Figure S4 showed the Zeta potentials of +23.2 , $-14.2,-16.2,5.9,-22.8,-10.9$ and $+4.7 \mathrm{mV}$, for $\mathrm{Au}$ nanorods, JGMSNs, JGMSNs (CTAB free), JGMSNs$\mathrm{NH}_{2}$, JGMSNs - $\mathrm{COOH}$, FA-JGMSNs and FA-JGMSNsBer respectively. The Ber loading efficiency and the Ber loading content on JGMSNs were respectively $47.4 \%$ and $19.1 \%$. These FA-JGMSNs-Ber exhibited no obvious change in size and PDI (Figure S5) after 1-week storage period in water. Then, we investigated the Ber release behavior of Ber-loaded FA-JGMSNs (FA-JGMSNs-Ber) in PBS at different $\mathrm{pH}$ values (7.4 and 5.5). As shown in Figure 1D, JGMSNs-Ber exhibited a pH-responsive drug release fashion. The cumulative content of releasing Ber was less than $7 \%$ at $\mathrm{pH} 7.4$ after 96 hours, whereas $50 \%$ of Ber was released after 96 hours at $\mathrm{pH}$ 5.5. This $\mathrm{pH}-$ responsive Ber release property, which resulted from the protonation and dissociation in acidic environments, was favorable for Ber release in tumor tissues rather than normal tissues since both the microenvironments of extracellular tumor tissue and intracellular endo/lysosomes were acidic.

To verify the FA-targeting capacity of FA-JGMSNs on liver cancer cells, JGMSNs and FA-JGMSNs were respectively labeled with the fluorescence marker FITC and their cellular internalization were quantified by flow cytometry. As shown in Figure 2A, the fluorescence intensity in FA-JGMSNs -treated group was significantly higher than that in JGMSNstreated group in FA receptor-positive SMMC-7721 cells. Moreover, preincubating free FA could effectively reduce the fluorescence sign of FA-JGMSNs due to the competitive blocking of the FA receptor. On the contrary, FA-JGMSNs and JGMSNs showed similar cellular internalization in the HL-7702 cells and both of their uptake in HL-7702 cells were lower than that in SMMC-7721 cells. Additionally, there was 

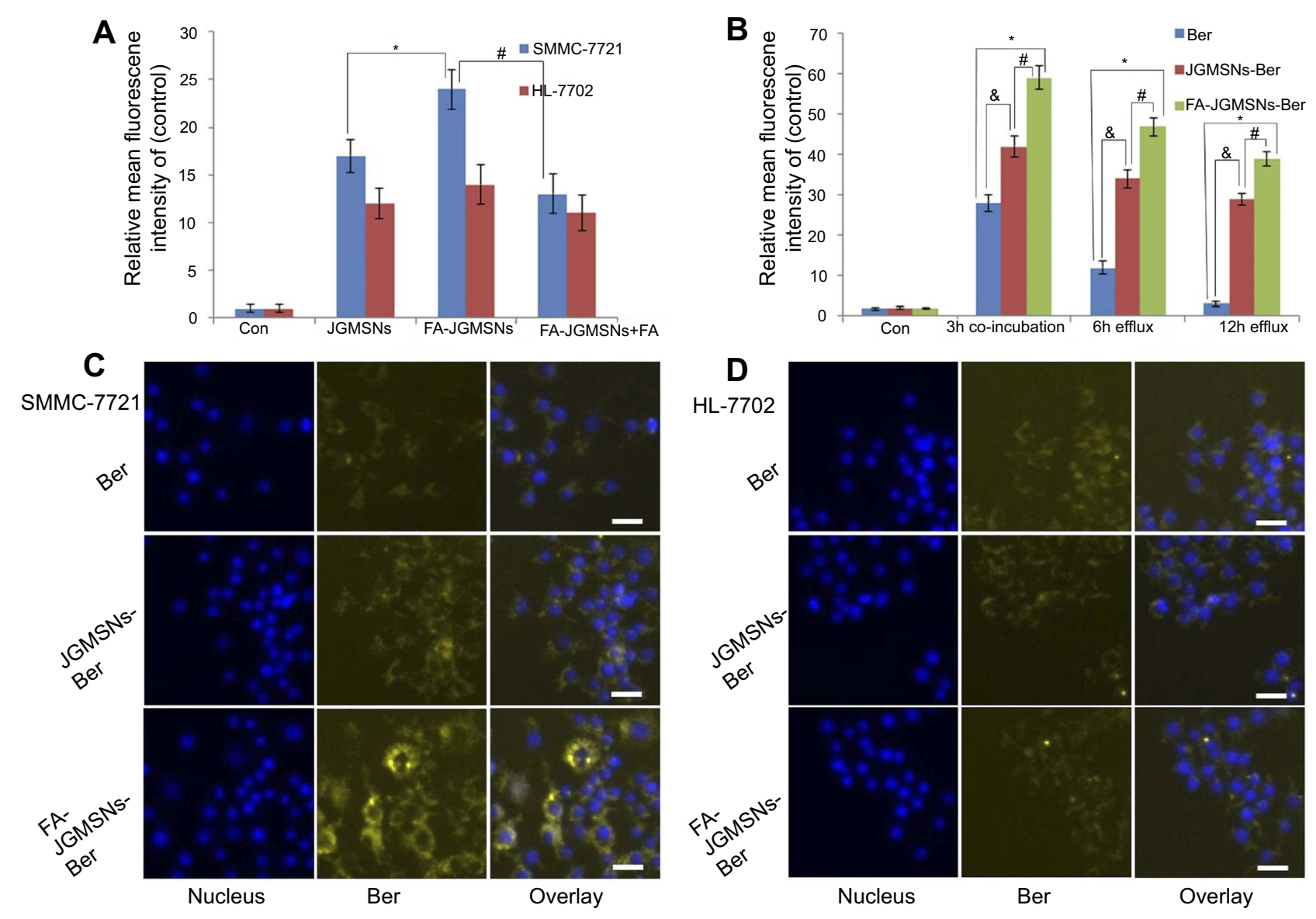

Figure 2 The delivery of Ber into cells. (A) The uptake of the FITC-labeled JGMSNs or FA-JGMSNs in the SMMC-772I cells and HL-7702 cells after 3 hours of incubation by FACS. All the data represented three separate experiments and are presented as the mean values \pm SD, and $* P<0.05$ represented JGMSNs group vs the FA-JGMSNs group and ${ }^{\#} P<0.05$ represented FA-JGMSNs group vs the FA-JGMSNs+FA group. (B) The fluorescence intensity of Ber in SMMC-772I after co-incubation with free Ber, JGMSNsBer, or FA-JGMSNs-Ber for 3 hours and then excreting Ber after another 6 or 12 hours by flow cytometry. ${ }^{* P}<0.05$ vs the FA-JGMSNs-Ber group; ${ }^{\#} P<0.05$ represents the JGMSNs-Ber group vs the FA-JGMSNs-Ber group; ${ }^{\&} P<0.05$ represents the Ber group vs the JGMSNs-Ber group. Confocal microscopy images of Ber distribution in (C) SMMC-772I cells or (D) HL-7702 after 3 hours of co-incubation with free Ber, JGMSNs-Ber or FA-JGMSNs-Ber; scale bars represent 100 um.

no significant influence on the uptake of FA-JGMSNs in HL7702 cells when free FA was applied. These results demonstrated the FA-targeted endocytosis of FA-JGMSNs in liver cancer cells. Then, we explored the targeted delivery of Ber capacity by observing intracellular distribution of Ber in SMMC-7721 cells and HL-7702 cells. CLSM imaging in Figure $2 \mathrm{C}$ and $\mathrm{D}$ showed that more fluorescence signals from Ber were observed in SMMC-7721 cells after being treated with JGMSNs-Ber than those treated with free Ber for 3 hours, which implied that JGMSNs-Ber could deliver Ber into cells and release Ber to the cytoplasm. Additionally, the FA-JGMSNs-Ber group exhibited the most yellow fluorescence signals in the cytoplasm of SMMC-7721 cells, which was due to the enhanced cell internalization by FA-targeted endocytosis. On the contrary, there was no obvious difference of fluorescence signals between the FA-JGMSNs-Ber group and the JGMSNs-Ber group in HL-7702 cells. Both of the yellow fluorescence intensities in the HL-7702 cells were remarkably weaker than those in SMMC-7721 cells. These selective distributions of Ber in cancer cells and normal cells originated from selective endocytosis as well as $\mathrm{pH}-$ responsive drug release, which were conducive to achieving an improved therapeutic effect of Ber. Considering that the main factor of chemotherapeutic resistance is the rapid pumpout by p-glycoprotein, ${ }^{45,46}$ we quantified the distribution of Ber in SMMC-7721 cells after different incubation times and evaluated the efflux of Ber after another 6 or 12 hours by flow cytometry. As shown in Figure 2B, FA-JGMSNs-Ber and JGMSNs-Ber could effectively enhance the Ber accumulation compared with free Ber in SMMC-7721 cells and FAJGMSNs-Ber showed better performance than JGMSNs-Ber, which were in line with CLSM results. Then, the cells were washed and allowed to excrete Ber for another 6 or 12 hours. The mount of Ber in the free Ber-treated group drastically decreased after 6 hours efflux and were negligible after 12 hours efflux. Interestingly, the fluorescence signals of Ber 
could still be detected in both the FA-JGMSNs-Ber-treated group and the JGMSNs-Ber-treated group after 12 hours efflux, indicating these nanocarriers could increase Ber retention in cancer cells. The FA-JGMSNs could effectively surmount the chemotherapeutic resistance of pump efflux, which contributed to further improving the chemotherapeutic effect.

To verify the therapeutic effect of FA-JGMSNs-Ber, SMMC-7721 cells and HL-7702 cells were co-incubated with JGMSNs, FA-JGMSNs, free Ber, JGMSNs-Ber or FA-JGMSNs-Ber for 24 hours and their cytotoxicity was measured by SRB assay. As illustrated in Figure 3A and B, the cell viability was still above $90 \%$ in the FA-JGMSNstreated group or the JGMSNs-treated group when the concentration of nanoparticles reached $10 \mu \mathrm{g} / \mathrm{mL}$, indicating that these nanocarriers possessed excellent biocompatibility. Free Ber, JGMSNs-Ber and FA-JGMSNs-Ber all exhibited the dose-dependent cytotoxicity towards
SMMC-7721 cells and HL-7702 cells. JGMSNs-Ber killed more SMMC-7721 cells than the equal dose of free Ber and FA-JGMSNs-Ber showed the best anti-cancer effect. Conversely, more HL-7702 cells lived when treated with JGMSNs-Ber or FA-JGMSNs-Ber compared with those treated with free Ber. The selective kill effect was consistent with Ber intracellular distributions. Encouraged by the excellent chemotherapeutic effect of FA-JGMSNs-Ber, we further investigated the combined chemo-radiotherapeutic effect based on FA-JGMSNs-Ber. As illustrated in Figure $3 \mathrm{C}$, the radiotherapeutic effect to SMMC-7721 cells was substantially improved after being treated with FAJGMSNs or JGMSNs compared with those without any treatment of nanoparticles, demonstrating the radiosensitization of these nanoparticles, which was in line with our previous reports. ${ }^{31}$ Additionally, JGMSNs-Ber with X-ray irradiation killed more SMMC-7721 cells
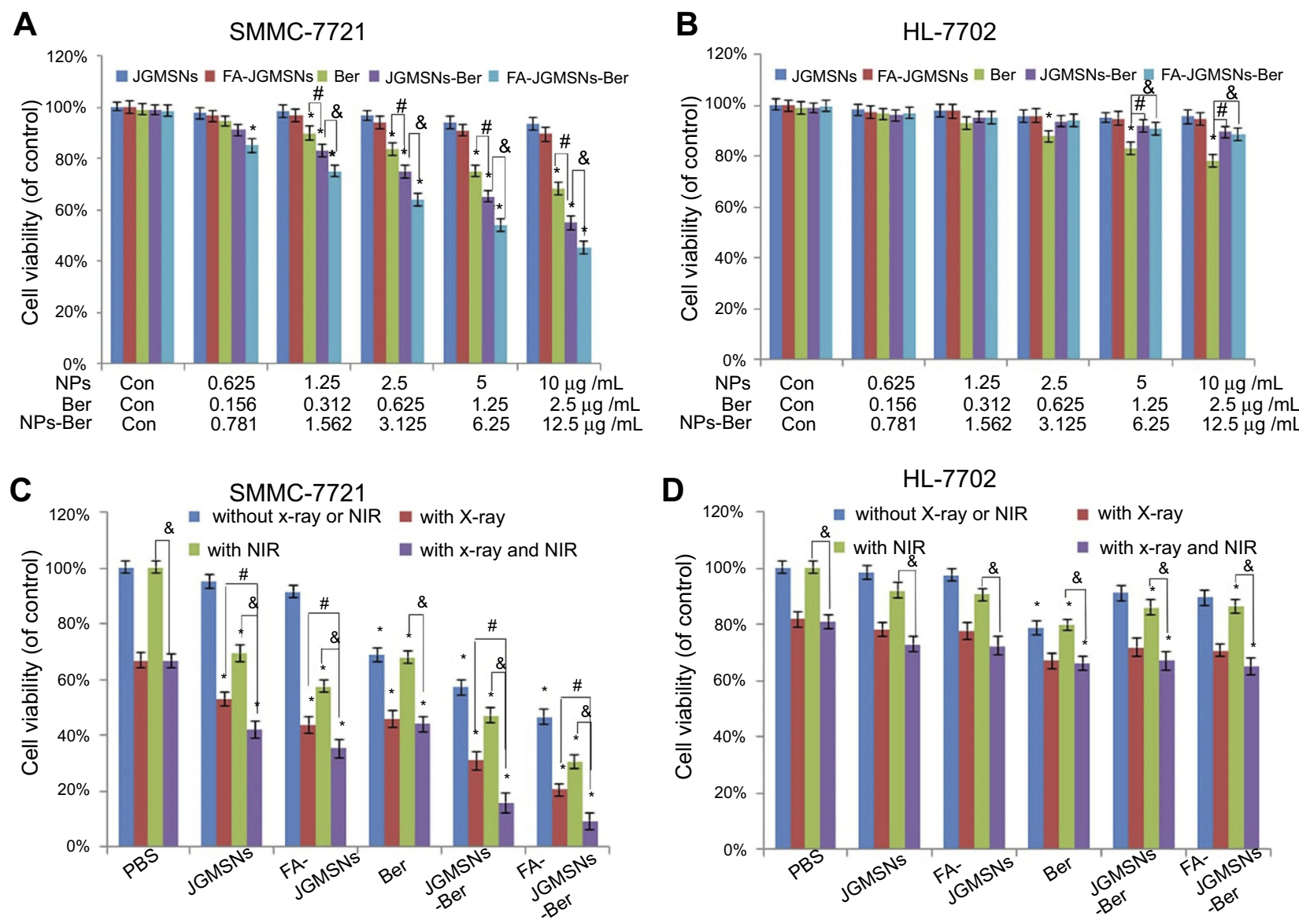

Figure 3 Cytotoxicity of FA-JGMSNs-Ber: The cytotoxicity of JGMSNs, FA-JGMSNs, free Ber, JGMSNs-Ber, and FA-JGMSNs-Ber against SMMC-772I cells (A) and HL-7702 cells (B) at various concentrations for 24 hours. (NPs represent JGMSNs and FA-JGMSNs; NPs-Ber represent JGMSNs-Ber and FA-JGMSNs-Ber). The data were three separate experiments. Mean values \pm SD, and $* P<0.05$ represented the tagged group vs the control group, ${ }^{\#} P<0.05$ represents the Ber group vs the JGMSNs-Ber group, and ${ }^{\&} P<0.05$ represents the JGMSNs-Ber group vs the FA-JGMSNs-Ber group. The cell viabilities of SMMC-772I cells (C) and HL-7702 cells (D) after being treated with PBS, JGMSNs, FA-JGMSNs, free Ber, JGMSNs-Ber or FA-JGMSNs-Ber with or without NIR and the absence or presence of RT. $* P<0.05$ represents the tagged group vs the control group, " $P<0.05$ represents the "with the X-ray" group vs "with the X-ray and NIR" group, and " $P<0.05$ represents the "with the NIR" group vs "with the X-ray and NIR" group. 
than either JGMSNs-Ber without RT or JGMSNs with RT and FA-JGMSNs-Ber with RT showed better therapeutic effect than JGMSNs-Ber with RT. Some reports have revealed that the combination of chemotherapy with RT could exert excellent synergistic therapeutic effect because chemotherapy could effectively inhibit the repair of radiation-induced DNA damage as well as arrest cell cycle in the radiosensitive $\mathrm{G} 2 / \mathrm{M}$ phase. ${ }^{4,48}$ Moreover, the combination of chemotherapy with RT could lead to an endoplasmic reticulum stress in tumor tissues, which induced the autophagy and apoptosis of cancer cells and the release of ATP and high mobility group box 1 (HMGB1) as well as the intratumoral accumulation of dendritic cells, $\gamma \delta$ $\mathrm{T}$ cells and CD8+ T lymphocytes, resulting in an immune response for enduring the powerful anti-tumor effect. ${ }^{49}$ Our results confirmed the high efficiency of chemoradiotherapy by FA-JGMSNs-Ber, which were consistent with previously reported applications of nanoparticles for combined chemoradiotherapy. ${ }^{50,51}$ Notably, FA-JGMSNsBer combining with RT didn't obviously increase the mortality of HL-7702 cells compared with RT (Figure 3D). It was due to the weak uptake of FA-JGMSNs-Ber and selective response Ber release as well as the low toxicity of Ber.

For further expanding the anti-tumor effect, PTT was combined with chemo-radiotherapy. We firstly explored the photothermal conversion efficiency of FA-JGMSNs-Ber in culture medium. As shown in Figure S6, only a $5^{\circ} \mathrm{C}$ increase was measured in pure water and free Ber solution as well as the cell medium under the near infrared (NIR) irradiation, whereas the temperature increases of FAJGMSNs-Ber solution exhibited a time- and concentrationdependent fashion. The temperature reached $43^{\circ} \mathrm{C}$ when the concentration of FA-JGMSNs-Ber was $12.5 \mu \mathrm{g} / \mathrm{mL}$ under the NIR irradiation of less than 5 minutes, which was up to the criteria of inducing cancer cells death. Then, we explored the localized photothermal effect and evaluated the triple-model therapies in vitro. As shown in Figure $3 \mathrm{C}$, the viability of SMMC-7721 cells only under NIR irradiation without any treatment was reduced by $<5 \%$, indicating the NIR irradiation was safe for the SMMC7721 cells. When pre-treated with JGMSNs or FAJGMSNs, the NIR irradiation led to a substantial toxicity towards SMMC7721 cells, demonstrating the effectiveness of PTT by these nanoparticles. Interestingly, the NIR irradiation combining with $\mathrm{X}$-ray irradiation caused more deaths of cells than either NIR irradiation or X-ray irradiation after treatment with JGMSNs or FA-JGMSNs.
Additionally, JGMSNs-Ber or FA-JGMSNs-Ber with NIR irradiation showed stronger killing effects in comparison to those without NIR irradiation. These results indicated the anticancer effects of the combination treatment of RT with PTT or chemotherapy with PTT were better than single PTT, RT or chemotherapy. As we expected, triple-model therapies based on FA-JGMSNs-Ber exhibited the best therapeutic effect. The synergistic effect probably originated from the increased temperatures improving the sensitivity of the cancer cells to RT and chemotherapy. Furthermore, over $90 \%$ of HL-7702 cells survived after treatment with nanoparticles under NIR owing to the better hyperthermia tolerance of normal cells in Figure 3D. Notably, chemo/ radio/photothermal therapeutics didn't cause the amounts of deaths of HL-7702 cells, indicating an excellent biosafety of the triple-model therapy.

To further verify the triple-model therapy in vivo, nude mice SMMC-7721 xenograft models were established. Then, PBS, free Ber, FA-JGMSNs, JGMSNs-Ber and FAJGMSNs-Ber were respectively administered into the mice every 3 days. In X-ray irradiation groups (RT, FAJGMSNs + RT, FA-JGMSNs-Ber + RT, JGMSNs-Ber + RT + NIR, FA-JGMSNs-Ber + RT+NIR), the mice received 5 Gy of X-ray radiation. In the NIR irradiation groups (JGMSNs-Ber + RT + NIR, FA-JGMSNs-Ber + RT + NIR), the tumor sites were exposed under an $808 \mathrm{~nm}$ laser for 5 minutes. As illustrated in Figure 4A-C, free Ber couldn't inhibit tumor growth compared with the PBS group due to its poor bioavailability. FA-JGMSNs-Ber exhibited a substantial anti-tumor effect, indicating that FA-JGMSNs were effective nanocarriers for improving therapeutic effect of Ber. Additionally, FA-JGMSNs + RT performed better than RT, demonstrating that FAJGMSNs were also effective radiosensitizers. Furthermore, FA-JGMSNs-Ber combining with RT displayed higher tumor inhibition rates than FA-JGMSNsBer or FA-JGMSNs with RT, confirming the combined effect by FA-JGMSNs-Ber. As we expected, when NIR was administrated, the therapeutic efficiency of FAJGMSNs-Ber with RT were remarkably enhanced. Moreover, FA-JGMSNs-Ber + RT + NIR showed the higher level of tumor-growth inhibition than JGMSNsBer + RT + NIR. These findings revealed the excellent anti-tumor effect of the triple-model therapy by FAJGMSNs-Ber. To further detect the systemic toxicity of FA-JGMSNs-Ber, we measured the blood chemistry indexes (liver function indexes: ALT, AST and kidney function indexes: CRE, BUN) after various treatments. 

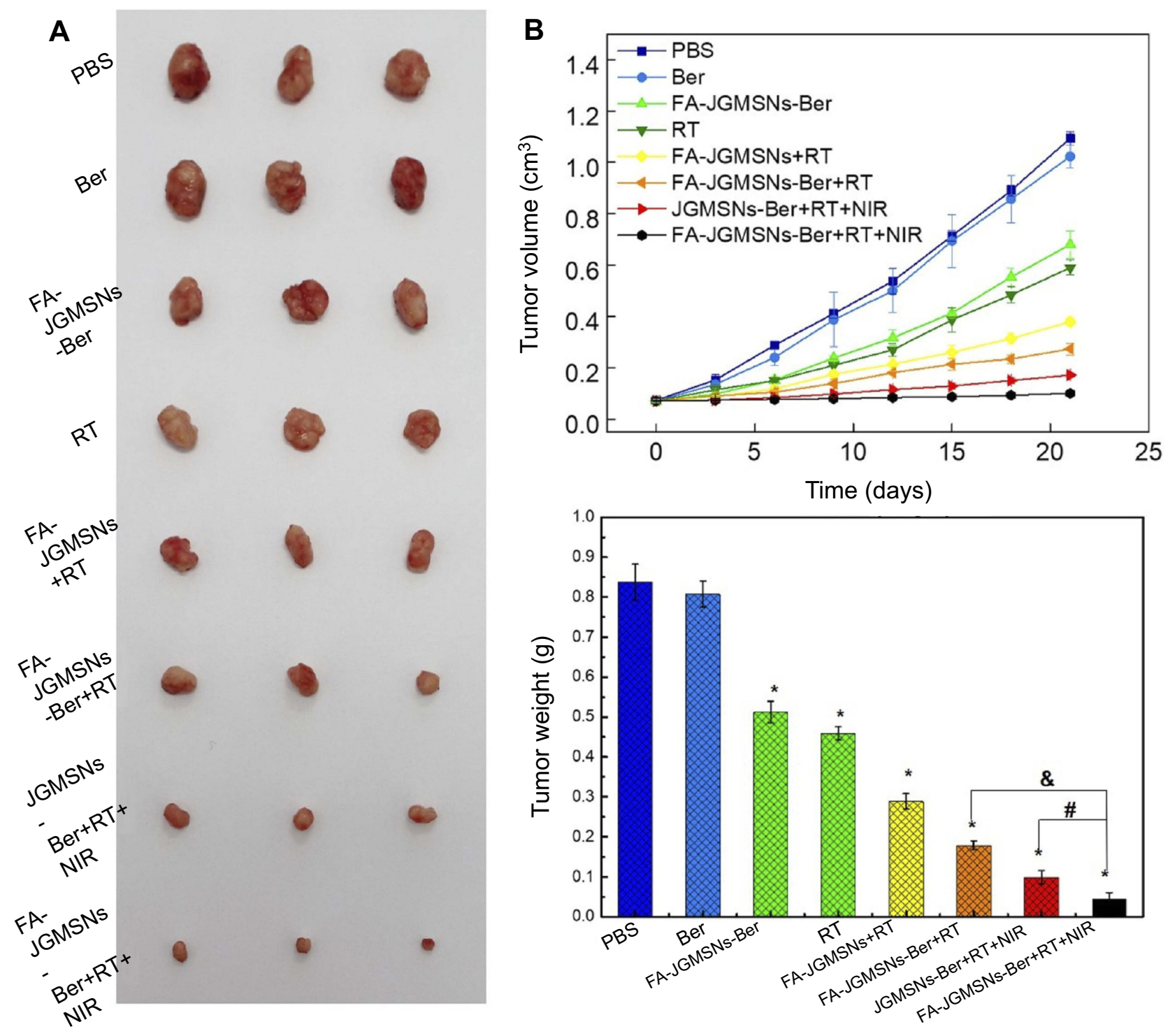

Figure 4 In vivo anti-tumor effect: $(\mathbf{A})$ tumor photographs, $(\mathbf{B})$ tumor growth curves, and $(\mathbf{C})$ tumor weights and inhibition rates of each group. $(* P<0.05$ represents the tagged group vs the PBS group. ${ }^{\#} P<0.05$ represents JGMSNs-Ber+RT+NIR group vs the FA-JGMSNs-Ber+RT+NIR group, and ${ }^{\&} P<0.05$ represents FA-JGMSNs-Ber+RT group vs the FA-JGMSNs-Ber+RT+NIR group).

As illustrated in Figure 5A-D, the levels of ALT, AST, CRE, and BUN were not significantly changed in all the groups compared with control groups. The good liver and kidney functions indicated an excellent biosafety of FAJGMSNs-Ber.

Radiation-induced intestinal injury is a severe clinical problem in patients after RT. The protective efficacy of Ber against radiation-induced intestinal injury has been demonstrated by several studies. ${ }^{52,53}$ Based on the higher bioavailability of FA-JGMSNs-Ber, we deemed that FAJGMSNs-Ber could preferably attenuate the radiationinduced intestinal syndrome. To validate our hypothesis, we administrated the fractionated X-ray irradiation to the xenografts of nude mice bearing SMMC-7721 cells after treatments with PBS, FA-JGMSNs, free Ber or FAJGMSNs-Ber. After courses of treatment, we detected the body weight and food intake of these mice. As shown in Figure $6 \mathrm{~A}$ and $\mathrm{B}$, the decrease of body weights and average chow intake showed radiation dose-dependent in RT groups and FA-JGMSNs+RT groups. When treated with free Ber, the decrease of body weights and average chow intake were relieved. More importantly, there was no observable body weight losses or chow intake decrease in the FA-JGMSNs-Ber + RT groups compared with the PBS-treated groups, which was better performance than that in free Ber groups. These results demonstrated that 

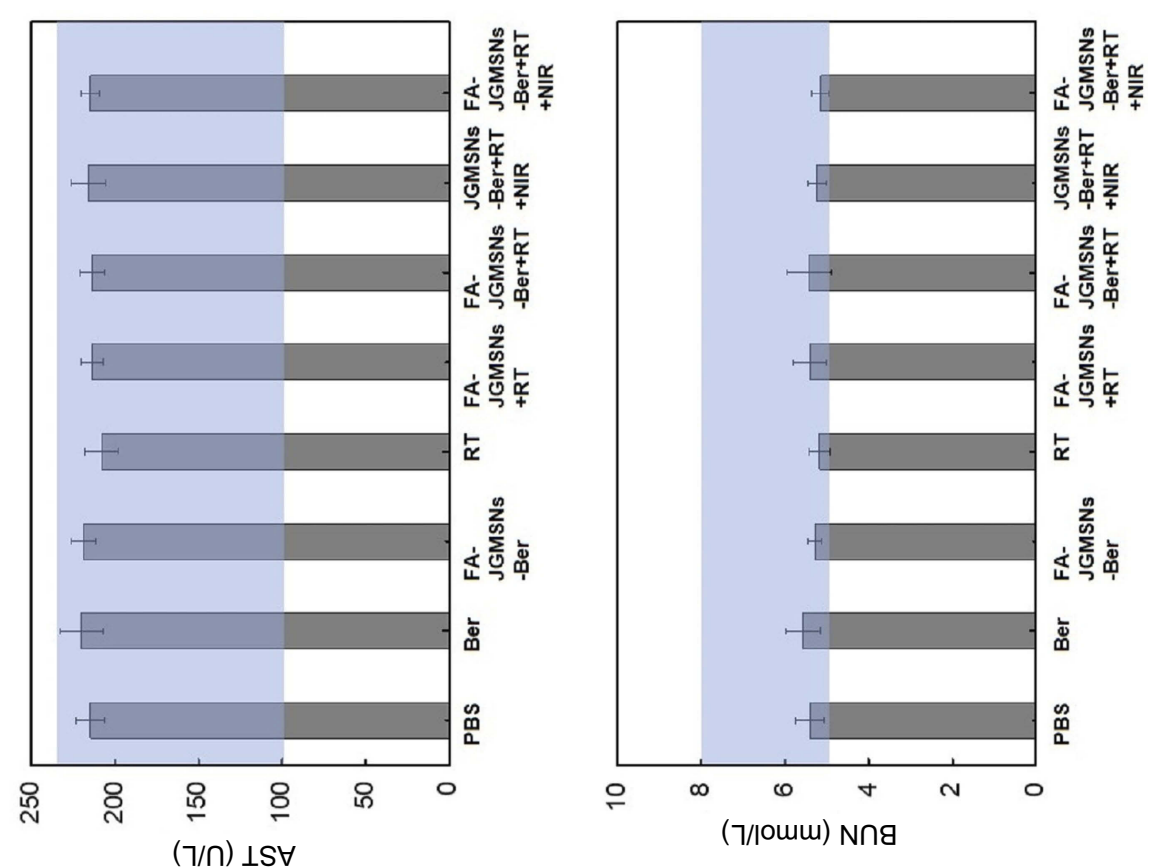

$\boldsymbol{m}$

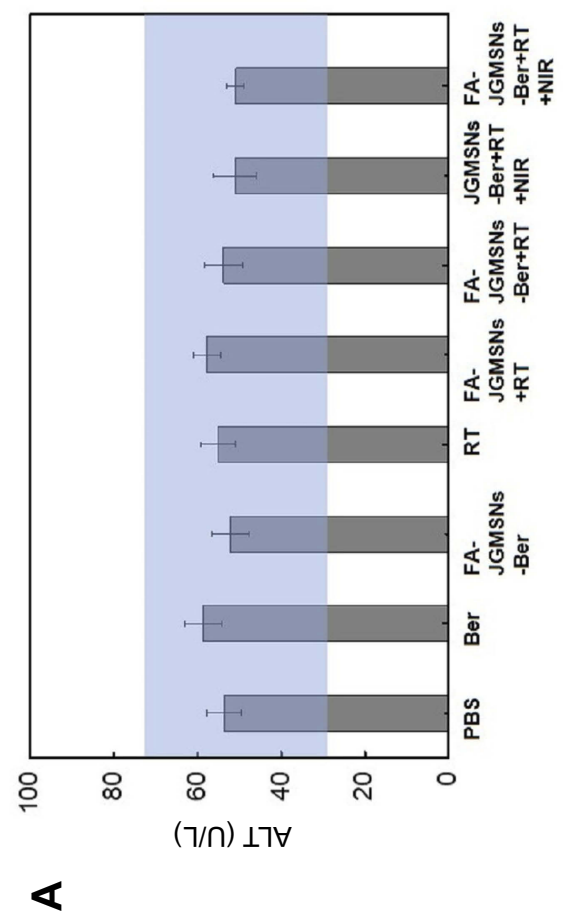

อ

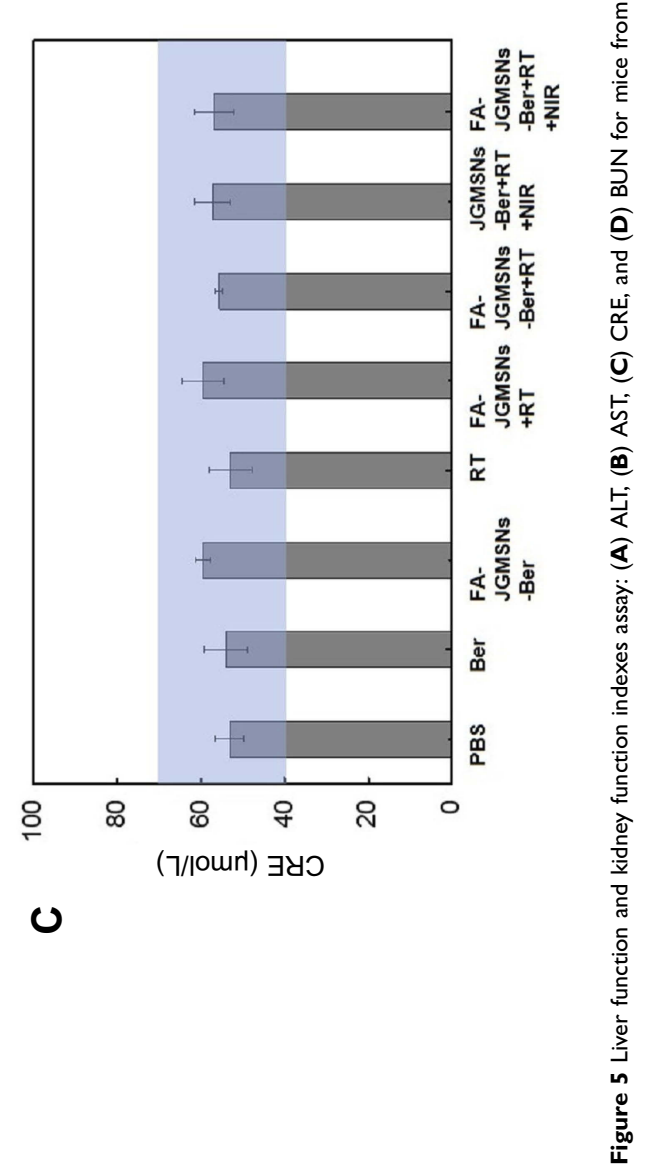



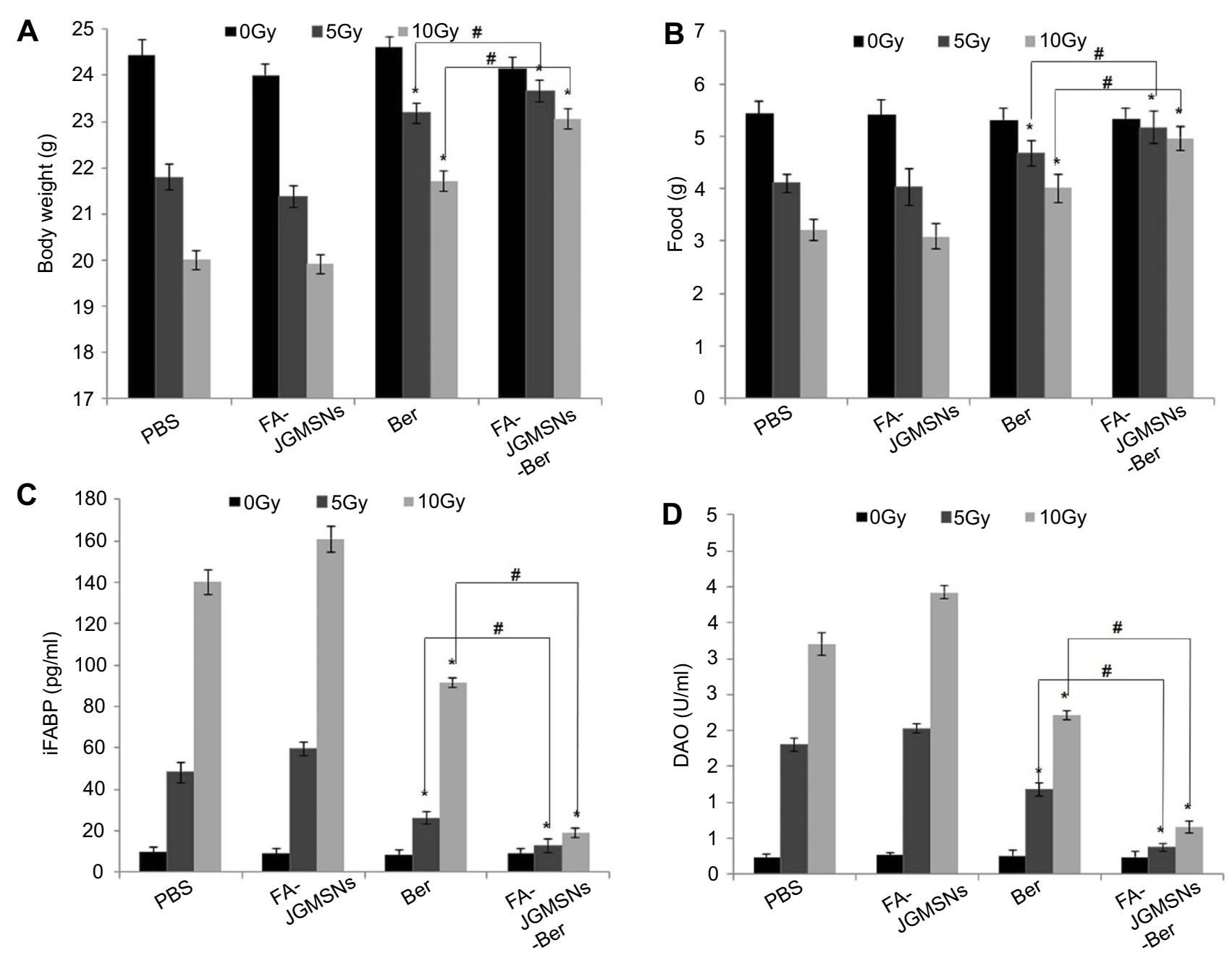

Figure 6 The protective effect against radiation-induced intestinal injury (A) body weight, (B) food intake, (C) diamine oxidase (DAO), and (D) intestinal fatty acid binding protein (iFABP) levels in plasma of mice after treatment with PBS, FA-JGMSNs, free Ber or FA-JGMSNs-Ber with or without RT. $* P<0.05$ represents the tagged group vs the PBS group, and ${ }^{\#} P<0.05$ represents the Ber group vs the FA-JGMSNs-Ber group.

FA-JGMSNs-Ber possessed excellent capacities to maintain the body weight and food intake of the mice receiving RT. Furthermore, we detected the level of iFABP and DAO in the plasmid to evaluate the early phases of intestinal mucosal injury of these mice. As shown in Figure 6C and $\mathrm{D}$, the levels of iFABP and DAO in the plasmid were very low without RT because iFABP and DAO were limited in the intestine site. After the X-ray irradiation, the level of iFABP and DAO in the PBS-treated, FAJGMSNs-treated and Ber-treated groups increased with the improving of radiation dose, which was due to the damage of intestinal mucosal by RT. Furthermore, Ber significantly delayed the increasing of iFABP and DAO. Encouragingly, FA-JGMSNs-Ber group exhibited the lower level of iFABP and DAO compared with the free Ber groups. These findings indicated that FA-JGMSNs-Ber possessed an excellent efficacy to reduce radiation-induced intestinal mucosal injury triggered by RT.

\section{Conclusion}

In summary, an FA-targeting Janus gold mesoporous silica nanocarriers (FA-JGMSNs) were fabricated to preload Ber for chemo-radio-photothermal therapy of liver cancer. On one hand, FA-JGMSNs showed a high Ber loading capacity and a tumor microenvironment-responsive Ber release behavior as well as a prolonged Ber intracellular retention, thus improving the bioavailability of Ber and expanding its therapeutic efficiency. On the other hand, FA-JGMSNs were developed as dual-functional agents for radiation sensitization and photothermal therapy. FA-JGMSNs-Ber showed excellent anti-tumor e $\square$ cacy of combination therapies with less side-effects in in-vitro and in-vivo 
experiments. More importantly, FA-JGMSNs-Ber effectively relieved radiation-induced intestinal injury. Hence, we believed that FA-JGMSNs-Ber integrating highly efficient treatment of cancer tissue with the protection of normal tissue would be a promising candidate for multidimensional therapies of liver cancer.

\section{Acknowledgments}

This work was supported by the National Key R\&D Program of China (Grant no. 2017YFF0108600, 2017YFC0211900 and 2016YFF0103800), the National Natural Science Foundation of China (Grant no. 81771982, 61535010, 8160071152 and 21803075), Key Research Program of the Chinese Academy of Sciences (No. KFZD-SW-210), the Natural Science Foundation of Jiangsu Province (No. BE2015601), and the Natural Science Foundation of Jiangsu Province (Grant no BK20181236).

\section{Disclosure}

The authors report no conflicts of interest in this work.

\section{References}

1. Maluccio MM, Covey AM. Recent progress in understanding, diagnosing, and treating hepatocellular carcinoma. CA Cancer J Clin. 2012;62(6):394-399. doi:10.3322/caac.21161

2. Yang JD, Roberts LR. Hepatocellular carcinoma: a global view. Nature Rev Gastroenterol Hepatol. 2010;7(8):448-458. doi:10.1038/ nrgastro.2010.100

3. Bruix J, Gores GJ, Mazzaferro V. Hepatocellular carcinoma: clinical frontiers and perspectives. Gut. 2014;63(5):844-855. doi:10.1136/ gutjnl-2013-306627

4. Cheng JC, Chuang VP, Cheng SH, et al. Local radiotherapy with or without transcatheter arterial chemoembolization for patients with unresectable hepatocellular carcinoma. Int $J$ Radiat Oncol Biol Phys. 2000;47(2):435-442. doi:10.1016/S0360-3016(00)00462-4

5. Chen SW, Lin LC, Kuo YC, Liang JA, Kuo CC, Chiou JF. Phase 2 study of combined sorafenib and radiation therapy in patients with advanced hepatocellular carcinoma. Int J Radiat Oncol Biol Phys. 2014;88(5):1041-1047. doi:10.1016/j.ijrobp.2014.01.017

6. Knox JJ, Cleary SP, Dawson LA. Localized and systemic approaches to treating hepatocellular carcinoma. J Clin Oncol. 2015;33 (16): 1835-1844. doi:10.1200/JCO.2014.60.1153

7. Chu KF, Dupuy DE. Thermal ablation of tumours: biological mechanisms and advances in therapy. Nature Rev Cancer. 2014;14 (3):199-208. doi:10.1038/nrc3672

8. Hwang J, Chan D, Chang T, et al. Effects of oral arginine and glutamine on radiation-induced injury in the rat. $J$ Surg Res. 2003;109(2):149-154.

9. Hauerjensen M, Wang J, Boerma M, Fu Q, Denham JW. Radiation damage to the gastrointestinal tract: mechanisms, diagnosis, and management. Curr Opin Support Palliat Care. 2007;1(1):23-29. doi:10.1097/SPC.0b013e3281108014

10. Cheng A, Kang YK, Chen Z, et al. Efficacy and safety of sorafenib in patients in the Asia-Pacific region with advanced hepatocellular carcinoma: a phase III randomised, double-blind, placebo-controlled trial. Lancet Oncol. 2009;10(1):25-34. doi:10.1016/S1470-2045(08)70285-7
11. Gish RG, Porta C, Lazar L, et al. Phase III randomized controlled trial comparing the survival of patients with unresectable hepatocellular carcinoma treated with nolatrexed or doxorubicin. J Clin Oncol. 2007;25(21):3069-3075. doi:10.1200/JCO.2006.08.4046

12. Kraus A, Ferber I, Bachmann S, et al. In vitro chemo- and radio-resistance in small cell lung cancer correlates with cell adhesion and constitutive activation of AKT and MAP kinase pathways. Oncogene. 2002;21(57):8683-8695. doi:10.1038/sj.onc.1205939

13. Fukuda K, Hibiya Y, Mutoh M, Koshiji M, Akao S, Fujiwara H. Inhibition by berberine of cyclooxygenase- 2 transcriptional activity in human colon cancer cells. J Ethnopharmacol. 1999;66(2):227-233.

14. Yue J, Wang Z, Shao D, et al. Cancer cell membrane-modified biodegradable mesoporous silica nanocarriers for berberine therapy of liver cancer. RSC Adv. 2018;8(70):40288-40297. doi:10.1039/ C8RA07574C

15. Wang Z, Wang Y, Chang Z, et al. Berberine-loaded Janus nanocarriers for magnetic field-enhanced therapy against hepatocellular carcinoma. Chem Biol Drug Des. 2017;89(3):464-469. doi:10.1111/ cbdd. 12866

16. Li G, Wang D, Hu Y, et al. Berberine inhibits acute radiation intestinal syndrome in human with abdomen radiotherapy. Med Oncol. 2010;27(3):919-925. doi:10.1007/s12032-009-9307-8

17. Yang X, Yang B, Cai J, et al. Berberine enhances radiosensitivity of esophageal squamous cancer by targeting HIF-1 $\alpha$ in vitro and in vivo. Cancer Biol Ther. 2013;14(11):1068-1073. doi:10.4161/cbt.26426

18. Tan W, Li Y, Chen M, Wang Y. Berberine hydrochloride: anticancer activity and nanoparticulate delivery system. Int J Nanomedicine. 2011;6:1773-1777. doi:10.2147/IJN.S22683

19. Kumar A, Ekavali CK, Mukherjee M, Pottabathini R, Dhull DK. Current knowledge and pharmacological profile of berberine: an update. Eur $J$ Pharmacol. 2015;761:288-297. doi:10.1016/j. ejphar.2015.05.068

20. Pund S, Borade G, Rasve G. Improvement of anti-inflammatory and anti-angiogenic activity of berberine by novel rapid dissolving nanoemulsifying technique. Phytomedicine. 2014;21(3):307-314. doi:10.1016/j.phymed.2013.09.013

21. Hainfeld JF, Dilmanian FA, Slatkin DN, Smilowitz HM. Radiotherapy enhancement with gold nanoparticles. J Pharm Pharmacol. 2008;60(8):977-985. doi:10.1211/jpp.60.8.0005

22. Roa W, Zhang X, Guo L, et al. Gold nanoparticle sensitize radiotherapy of prostate cancer cells by regulation of the cell cycle. Nanotechnology. 2009;20(37):375101. doi:10.1088/0957-4484/20/ $37 / 375101$

23. Her S, Jaffray DA, Allen C. Gold nanoparticles for applications in cancer radiotherapy: mechanisms and recent advancements. $A d v$ Drug Deliv Rev. 2017;109:84-101. doi:10.1016/j.addr.2015.12.012

24. Zhou M, Zhao J, Tian M, et al. Radio-photothermal therapy mediated by a single compartment nanoplatform depletes tumor initiating cells and reduces lung metastasis in the orthotopic $4 \mathrm{~T} 1$ breast tumor model. Nanoscale. 2015;7(46):19438-19447. doi:10.1039/ c5 5 r04587h

25. Banu H, Sethi DK, Edgar A, et al. Doxorubicin loaded polymeric gold nanoparticles targeted to human folate receptor upon laser photothermal therapy potentiates chemotherapy in breast cancer cell lines. J Photochem Photobiol B-Biol. 2015;149:116-128. doi:10.1016/j. jphotobiol.2015.05.008

26. Hu R, Zheng M, Wu J, et al. Core-shell magnetic gold nanoparticles for magnetic field-enhanced radio-photothermal therapy in cervical cancer. Nanomaterials. 2017;7(5):111. doi:10.3390/nano7120458

27. Wang Z, Chang Z, Lu M, et al. Janus silver/silica nanoplatforms for light-activated liver cancer chemo/photothermal therapy. ACS Appl Mater Interfaces. 2017;9(36):30306-30317. doi:10.1021/ acsami.7b06446

28. Shao D, Li M, Wang Z, et al. Bioinspired diselenide-bridged mesoporous silica nanoparticles for dual-responsive protein delivery. $A d v$ Mater. 2018;30:1801198. 
29. Shakerizadeh A, Shiran M, Khoee S, Sharifi AM, Ghaznavi H, Khoei S. A new magnetic nanocapsule containing 5-fluorouracil: in vivo drug release, anti-tumor, and pro-apoptotic effects on CT26 cells allograft model. J Biomater Appl. 2014;29(4):548-556. doi:10.1177/0885328214536940

30. Shakerizadeh A, Khoee S, Shiran M, Sharifi AM, Khoei S. Synergistic effects of magnetic drug targeting using a newly developed nanocapsule and tumor irradiation by ultrasound on CT26 tumors in BALB/c mice. J Mater Chem B. 2015;3(9):1879-1887. doi:10.1039/C4TB01708K

31. Keshavarz M, Moloudi K, Paydar R, et al. Alginate hydrogel co-loaded with cisplatin and gold nanoparticles for computed tomography image-guided chemotherapy. J Biomater Appl. 2018;33 (2):161-169. doi:10.1177/0885328218782355

32. Alamzadeh Z, Beik J, Mahabadi VP, et al. Ultrastructural and optical characteristics of cancer cells treated by a nanotechnology based chemo-photothermal therapy method. $J$ Photochem Photobiol B-Biol. 2019;192:19-25. doi:10.1016/j.jphotobiol.2019.01.005

33. Beik J, Jafariyan M, Montazerabadi A, et al. The benefits of folic acid-modified gold nanoparticles in CT-based molecular imaging: radiation dose reduction and image contrast enhancement. Artif Cells Nanomed Biotechnol. 2017;46(8):1993-2001.

34. Zhang Z, Wang L, Wang J, et al. Mesoporous silica-coated gold nanorods as a light-mediated multifunctional theranostic platform for cancer treatment. Adv Mat. 2012;24(11):1418-1423. doi:10.1002 adma.201104714

35. Liu R, Zhang Y, Zhao X, Agarwal A, Mueller LJ, Feng P. pHresponsive nanogated ensemble based on gold-capped mesoporous silica through an acid-labile acetal linker. J Am Chem Soc. 2010;132 (5):1500-1501. doi:10.1021/ja907838s

36. Viveroescoto JL, Slowing II, Wu C, Lin VSY. Photoinduced intracellular controlled release drug delivery in human cells by gold-capped mesoporous silica nanosphere. J Am Chem Soc. 2009;131(10):3462-3463. doi:10.1021/ja900025f

37. Wang Z, Shao D, Chang Z, et al. Janus gold nanoplatform for synergetic chemoradiotherapy and computed tomography imaging of hepatocellular carcinoma. ACS Nano. 2017;11(12):12732-12741. doi:10.1021/acsnano.7b07486

38. Wang Z, Wang Y, Lu M, et al. Janus Au-mesoporous silica nanocarriers for chemo-photothermal treatment of liver cancer cells. RSC Adv. 2016;6(50):44498-44505. doi:10.1039/C6RA04183C

39. Neshastehriz A, Tabei M, Maleki S, Eynali S, Shakeri-Zadeh A. Photothermal therapy using folate conjugated gold nanoparticles enhances the effects of $6 \mathrm{MV}$ X-ray on mouth epidermal carcinoma cells. J Photochem Photobiol B. 2017;172:52-60. doi:10.1016/j. jphotobiol.2017.05.012

40. Ghaznavi H, Hosseini-Nami S, Kamrava SK, et al. Folic acid conjugated PEG coated gold-iron oxide core-shell nanocomplex as a potential agent for targeted photothermal therapy of cancer. Artif Cells Nanomed Biotechnol. 2018;46(8):1594-1604. doi:10.1080/ 21691401.2017.1384384
41. Beik J, Khademi S, Attaran N, et al. A nanotechnology-based strategy to increase the efficiency of cancer diagnosis and therapy: folate-conjugated gold nanoparticles. Curr Med Chem. 2017;24 (39):4399-4416. doi:10.2174/0929867324666170810154917

42. Mirrahimi M, Hosseini V, Kamrava SK, et al. Selective heat generation in cancer cells using a combination of $808 \mathrm{~nm}$ laser irradiation and the folate-conjugated Fe2O3@Au nanocomplex. Artif Cells Nanomed Biotechnol. 2018;46(sup1):241-253. doi:10.1080/ 21691401.2017.1420072

43. Montazerabadi A, Beik J, Irajirad R, et al. Folate-modified and curcumin-loaded dendritic magnetite nanocarriers for the targeted thermo-chemotherapy of cancer cells. Artif Cells Nanomed Biotechnol. 2019;47(1):330-340. doi:10.1080/21691401.2018.1557670

44. Wang Y, Shao D, Zhang L, et al. Gold nanorods-silica Janus nanoparticles for theranostics. Appl Phys Lett. 2015;106(17):173705. doi:10.1063/1.4919454

45. Wu Q, Yang Z, Nie Y, Shi Y, Fan D. Multi-drug resistance in cancer chemotherapeutics: mechanisms and lab approaches. Cancer Lett. 2014;347(2):159-166. doi:10.1016/j.canlet.2014.03.013

46. Mi Y, Lou L. ZD6474 reverses multidrug resistance by directly inhibiting the function of P-glycoprotein. Br J Cancer. 2007;97 (7):934. doi:10.1038/sj.bjc.6603985

47. Kvols LK. Radiation sensitizers: a selective review of molecules targeting DNA and non-DNA targets. J Nucl Med. 2005;46 (1): $187 \mathrm{~S}$

48. Lu Q-B. Molecular reaction mechanisms of combination treatments of low-dose cisplatin with radiotherapy and photodynamic therapy. $J$ Med Chem. 2007;50(11):2601-2604. doi:10.1021/ jm061416b

49. Mirrahimi M, Khateri M, Beik J, et al. Enhancement of chemoradiation by co-incorporation of gold nanoparticles and cisplatin into alginate hydrogel. J Biomed Mat Res Part B. 2019. doi:10.1002/ jbm.b.34356

50. Kumar R, Belz J, Markovic S, et al. Nanoparticle-based brachytherapy spacers for delivery of localized combined chemoradiation therapy. Int J Radiat Oncol Biol Phys. 2015;91(2):393-400. doi:10.1016/j.ijrobp.2014.10.041

51. Park J, Park J, Ju EJ, et al. Multifunctional hollow gold nanoparticles designed for triple combination therapy and CT imaging. J Controlled Release. 2015;207:77-85. doi:10.1016/j.jconrel.2015.04.007

52. Li G, Zhang Y, Tang J, et al. Effects of berberine against radiation-induced intestinal injury in mice. Int $J$ Radiat Oncol Biol Phys. 2010;77(5):1536-1544. doi:10.1016/j.ijrobp.2010. 02.062

53. $\mathrm{Gu} \mathrm{L}, \mathrm{Li} \mathrm{N}, \mathrm{Yu} \mathrm{W}$, et al. Berberine reduces rat intestinal tight junction injury induced by ischemia-reperfusion associated with the suppression of inducible nitric oxide synthesis. Am J Chin Med (Gard City NY). 2013;41(6):1297-1312. doi:10.1142/S019 2415X13500870 


\section{Supplementary Materials}

Tetrachloroauric acid $\left(\mathrm{HAuCl}_{4} \cdot 3 \mathrm{H}_{2} \mathrm{O}\right)$, cetyltrimethyl ammonium bromide (CTAB), tetraethyl orthosilicate (TEOS), Sulforhodamine B (SRB), Hoechst 33,258, 3-Aminopropyltriethoxysilane (APS), sodium borohydride $\left(\mathrm{NaBH}_{4}\right)$, and fluorescein isothiocyanate (FITC) were obtained from Sigma-Aldrich Inc (St. Louis, MO, USA). Sodium hydroxide $(\mathrm{NaOH})$, ammonium hydroxide $\left(\mathrm{NH}_{4}\right.$ $\mathrm{OH}, 28 \%$ ), ammonium Nitrate $\left(\mathrm{NH}_{4} \mathrm{NO}_{3}\right)$, anhydrous ethanol, hydrochloric acid and succinic anhydride were obtained from Beijing Chemical Reagent Co. (Beijing, People's Republic of China). Bernerine (>99\%) was purchased from Shanghai Hualan Chemical Co. (Shanghai, People's Republic of China). FA-PEG-NH2 (MW =3400) were purchased from Xi'an Ruixi (Xi'an, China). RPMI1640 medium was purchased from GIBCO (Carlsbad, CA, USA). Fetal bovine serum (FBS) were obtained from Beyotime Institute of Biotechnology (Jiangsu, People's Republic of China). Diagnostic kits for aspartate aminotransferase (AST), alanine aminotransferase (ALT), blood urea nitrogen (BUN), creatinine (CRE) were purchased from Nanjing Jiancheng Bioengineering Institute (Nanjing, People's Republic of China). DAO ELISA Kit and iFABP ELISA Kit were purchased from Shanghai Chunmai biotechnology co. (Shanghai, People's Republic of China). All reagents were commercially available products with analytical grade purity and used without further purification.

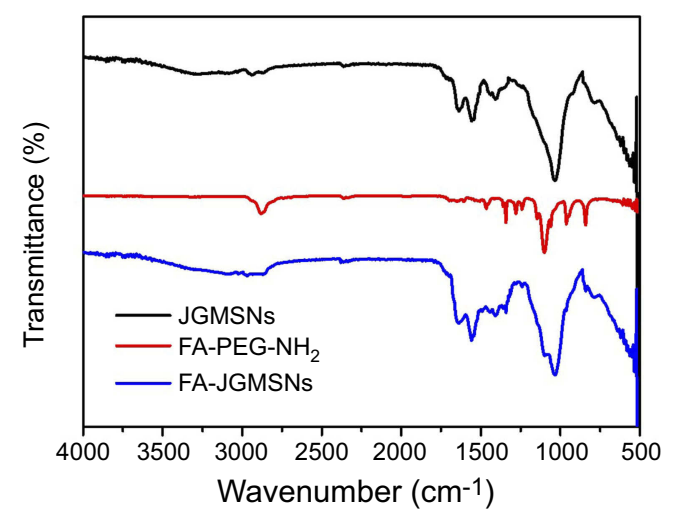

Figure SI FTIR spectra of JGMSNs, FA-PEG-NH 2 and FA-JGMSNs.

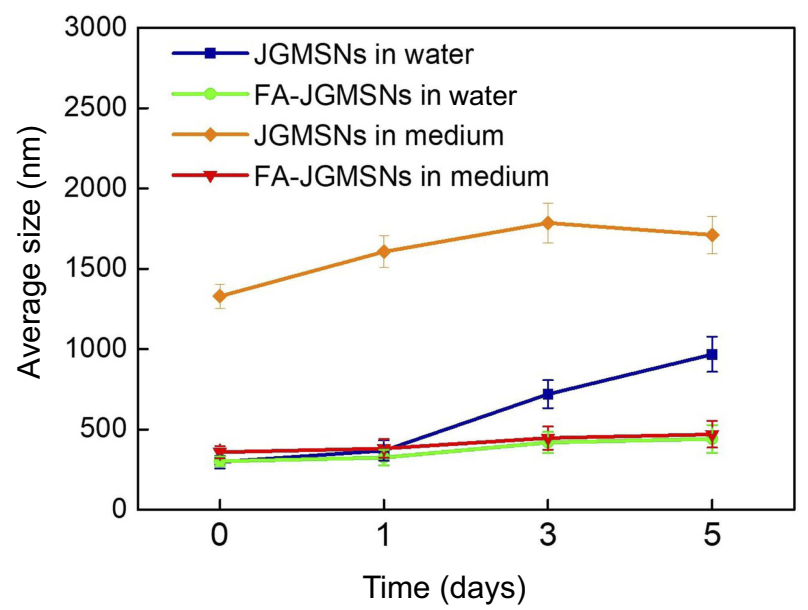

Figure S2 Average size of the FA-JGMSNs and JGMSNs in water and 1640 medium supplemented with I0\% of FBS for I, 3 and 5 days. These data represent three separate experiments and are presented as the mean values \pm SD. 


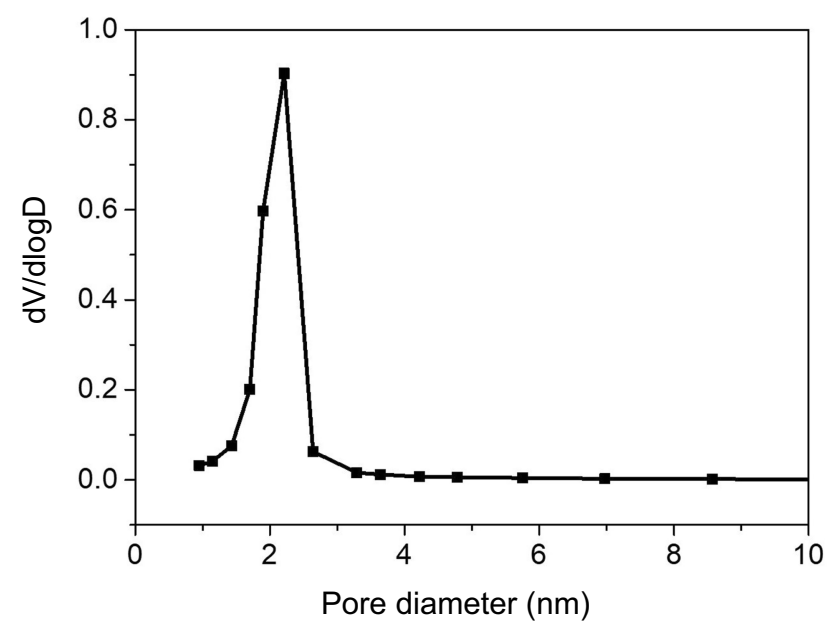

Figure S3 Pore size distribution of FA-JGMSNs.

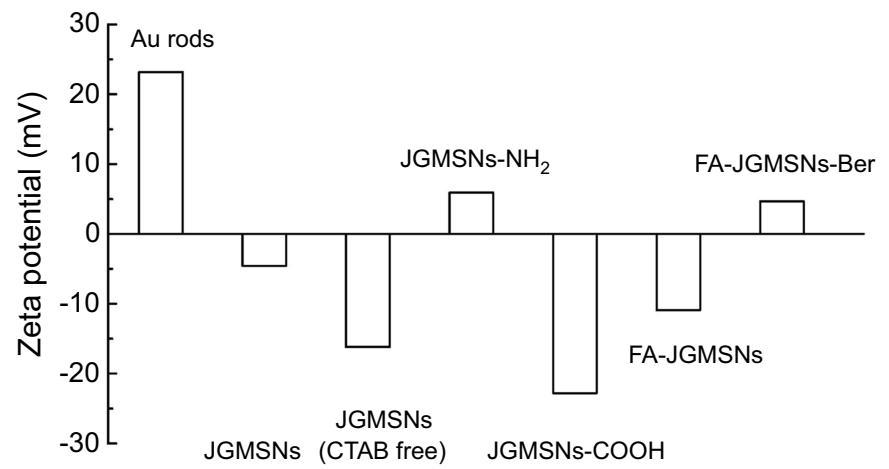

Figure S4 Zeta potential of these nanocomplexes in water.

A

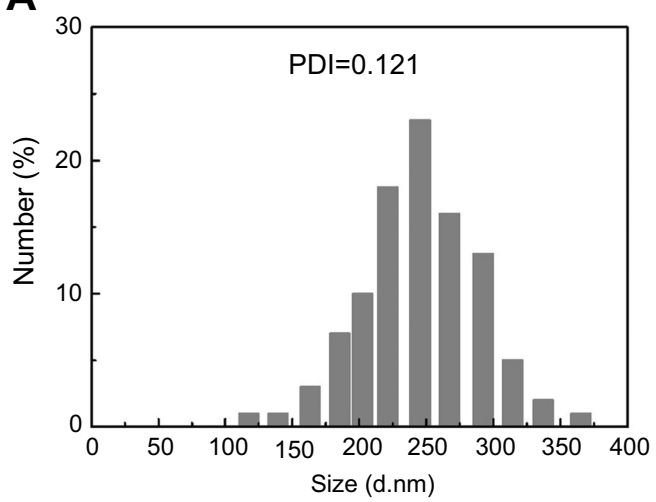

B

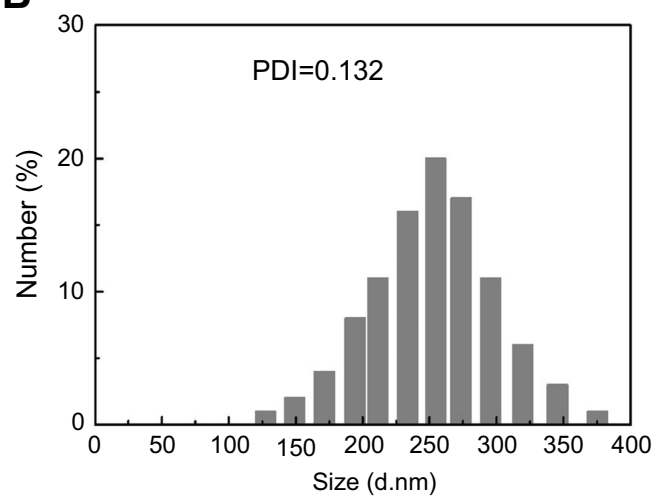

Figure S5 Size distribution of (A) FA-JGMSNs-Ber, and (B) FA-JGMSNs-Ber after 7-days storage in water. 


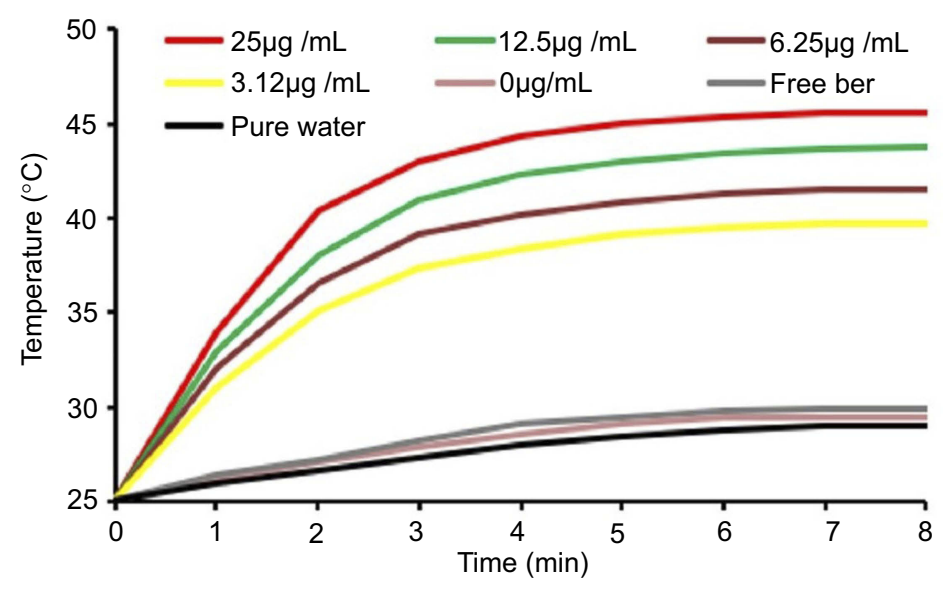

Figure S6 Temperature increase in FA-JGMSNs-Ber suspensions at various concentrations under NIR exposure.

\section{Publish your work in this journal}

The International Journal of Nanomedicine is an international, peerreviewed journal focusing on the application of nanotechnology in diagnostics, therapeutics, and drug delivery systems throughout the biomedical field. This journal is indexed on PubMed Central, MedLine, CAS, SciSearch ${ }^{\mathbb{B}}$, Current Contents ${ }^{\mathbb{B}} /$ Clinical Medicine,
Journal Citation Reports/Science Edition, EMBase, Scopus and the Elsevier Bibliographic databases. The manuscript management system is completely online and includes a very quick and fair peer-review system, which is all easy to use. Visit http://www.dovepress.com/ testimonials.php to read real quotes from published authors. 